\title{
Charm quark system in $2+1$ flavor lattice QCD using the PACS-CS configurations
}

PACS-CS Collaboration : Y. Namekawa ${ }^{\ddagger}$, S. Aoki ${ }^{b, c}$, N. Ishii ${ }^{a}$, K.-I. Ishikawa ${ }^{d}$, N. Ishizuka ${ }^{a, b}$, T. Izubuchi ${ }^{c, e}$, D. Kadoh ${ }^{a}$, K. Kanaya ${ }^{b}$, Y. Kuramashi ${ }^{a, b}$, M. Okawa $^{d}$, Y. Taniguchi ${ }^{a, b}$, A. Ukawa ${ }^{a, b}$, N. Ukita ${ }^{a}$, T. Yoshié ${ }^{a, b}$

${ }^{a}$ Center for Computational Sciences, University of Tsukuba, Tsukuba, Ibaraki 305-8577, Japan

${ }^{b}$ Graduate School of Pure and Applied Sciences, University of Tsukuba, Tsukuba, Ibaraki 305-8571, Japan

${ }^{c}$ Riken BNL Research Center, Brook-haven National Laboratory, Upton, New York 11973, USA

${ }^{d}$ Graduate School of Sciences, Hiroshima University, Higashi-Hiroshima, Hiroshima 739-8526, Japan

${ }^{e}$ Institute for Theoretical Physics, Kanazawa University, Kanazawa, Ishikawa 920-1192, Japan

\begin{abstract}
We study heavy-heavy and heavy-light quark systems for charm with a relativistic heavy quark action in 2+ 1 flavor lattice QCD. Configurations are generated by the PACS-CS Collaboration at the lattice spacing is $a=0.09 \mathrm{fm}$ with the lattice size of $32^{3} \times 64$ employing the $O(a)$-improved Wilson quark action and the Iwasaki gauge action. We present preliminary results for the charmonium spectrum and the $D$ and $D_{s}$ meson decay constants evaluated at $3.5 \mathrm{MeV}<m_{\mathrm{ud}}<12$ $\mathrm{MeV}$ with $m_{\mathrm{s}}$ around the physical value. We investigate the dynamical quark mass dependences of the hyperfine and the orbital splittings. The decay constants are compared with the recent experimental values.
\end{abstract}

The XXVI International Symposium on Lattice Field Theory

July 14-19 2008

Williamsburg, Virginia, USA

\footnotetext{
*Speaker.

${ }^{\dagger}$ E-mail: namekawa@ccs.tsukuba.ac.jp
} 


\section{Introduction}

Precise determination of physical quantities for heavy quark systems provides us with an opportunity to search for new physics beyond the standard model. For this purpose lattice QCD should be a powerful tool. However, the use of conventional lattice quark actions are problematic because of large cutoff errors due to the heavy quark masses. So far several approaches to avoid this problem have been employed for the study of heavy quark physics on the lattice[1]. Our choice is to employ the relativistic heavy quark action of Ref. [2]. This formalism allows us to take the continuum limit in which $m_{Q} a$ corrections are controlled by a smooth function. In fact the cutoff errors are reduced from $O\left(\left(m_{Q} a\right)^{n}\right)$ to $O\left(f\left(m_{Q} a\right)\left(a \Lambda_{Q C D}\right)^{2}\right)$ where $f\left(m_{Q} a\right)$ is an analytic function around $m_{Q} a=0$.

\section{Simulation parameters}

We simulate the charm quark system with the relativistic heavy quark action of Ref. [2] on the 2+1 flavor lattice QCD configurations which are generated by the PACS-CS Collaboration employing the nonperturbatively $O(a)$-improved Wilson quark action with $c_{\mathrm{SW}}^{\mathrm{NP}}=1.715[3]$ and the Iwasaki gauge action. The lattice size is $32^{3} \times 64$ whose spatial extent is $L=2.9 \mathrm{fm}$ with the lattice spacing of $a=0.09 \mathrm{fm}$. The dynamical up-down quark mass ranges from $67 \mathrm{MeV}$ down to 3.5 $\mathrm{MeV}$ which is close to the the physical value. The details of the configuration production and light hadron physics that emerges from them are described in Refs. [4, 5, 6, 7]. Table 1 summarizes the simulation parameters and the statistics of the configuration sets we have used for the heavy quark measurements. The number of the source points is to be quadrupled. We emphasize that the data point at $\left(\kappa_{\mathrm{ud}}, \kappa_{\mathrm{s}}\right)=(0.137785,0.13660)$ is almost on the physical point: the up-down quark mass is only $30 \%$ heavier and the strange quark mass is almost exactly at the physical value.

The relativistic heavy quark action proposed in Ref. [2] is given by

$$
\begin{aligned}
S_{Q}= & \sum_{x, y} \bar{Q}_{x} D_{x, y} Q_{y}, \\
D_{x, y}= & \delta_{x y}-\kappa_{\mathrm{h}} \sum_{i}\left[\left(r_{s}-v \gamma_{i}\right) U_{x, i} \delta_{x+\hat{i}, y}+\left(r_{s}+v \gamma_{i}\right) U_{x, i}^{\dagger} \delta_{x, y+\hat{i}}\right] \\
& -\kappa_{\mathrm{h}}\left[\left(r_{t}-v \gamma_{i}\right) U_{x, 4} \delta_{x+\hat{4}, y}+\left(r_{t}+v \gamma_{i}\right) U_{x, 4}^{\dagger} \delta_{x, y+\hat{4}}\right] \\
& -\kappa_{\mathrm{h}}\left[c_{B} \sum_{i, j} F_{i j}(x) \sigma_{i j}+c_{E} \sum_{i} F_{i 4}(x) \sigma_{i 4}\right],
\end{aligned}
$$

where we are allowed to choose $r_{t}=1$, while the other four parameters $v, r_{s}, c_{B}, c_{E}$ should be adjusted in the mass dependent way. We use the one-loop perturbative values for $r_{S}, c_{B}$ and $c_{E}$ evaluated in Ref. [8]. For the clover coefficients $c_{B}$ and $c_{E}$ we incorporate the nonperturbative contributions at the massless limit adopting the procedure $c_{B, E}=\left(c_{B, E}\left(m_{Q} a\right)-c_{B, E}(0)\right)^{\mathrm{PT}}+c_{\mathrm{SW}}^{\mathrm{NP}}$. At each simulation point, the parameter $v$ is nonperturbatively determined from the dispersion relation for the spin-averaged $1 S$ state of the charmonium:

$$
E(\vec{p})^{2}=E(\vec{p})^{2}+c_{\mathrm{eff}}^{2}|\vec{p}|^{2},
$$


Table 1: Simulation parameters. Quark masses are perturbatively renormalized in the $\overline{\mathrm{MS}}$ scheme. The renormalization scale is $\mu=1 / a$ for $\kappa_{\mathrm{ud}} \leq 0.137785$ and $\mu=2 \mathrm{GeV}$ for the physical point.

\begin{tabular}{ccccccc}
\hline$\kappa_{\mathrm{ud}}$ & $\kappa_{\mathrm{s}}$ & $m_{\mathrm{ud}}^{\overline{\mathrm{MS}}}(\mu)[\mathrm{MeV}]$ & $m_{\mathrm{s}}^{\overline{\mathrm{MS}}}(\mu)[\mathrm{MeV}]$ & \multicolumn{2}{c}{ \#conf } & \#source \\
& & & & measured & total/MD time & \\
\hline 0.13770 & 0.13640 & $12.3(2)$ & $90(1)$ & 400 & $800 / 2000$ & 1 \\
0.13781 & 0.13640 & $3.5(2)$ & $87(1)$ & 100 & $198 / 990$ & 1 \\
0.137785 & 0.13660 & $3.5(1)$ & $73(1)$ & 90 & $200 / 1000$ & 1 \\
\hline \multicolumn{2}{c}{ physical point } & $2.53(5)$ & $72.7(8)$ & & & \\
\hline
\end{tabular}

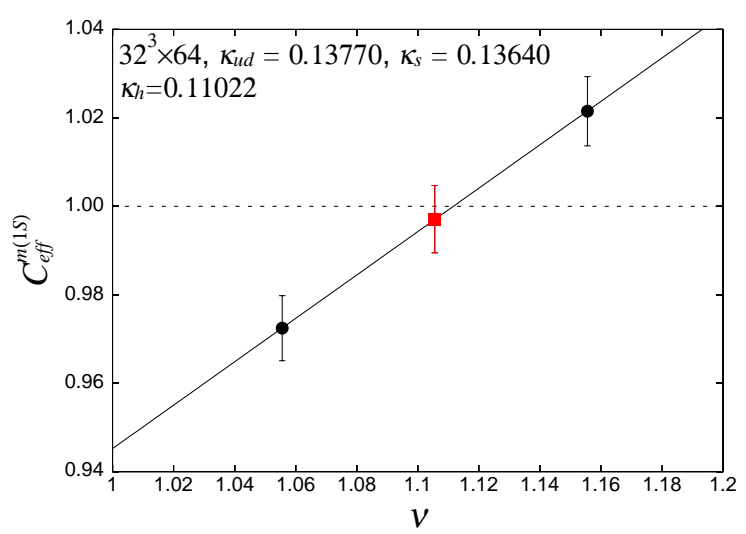

Figure 1: Nonperturbative tuning of $v$ with $\kappa_{\mathrm{h}}=0.11022$ at $\left(\kappa_{\mathrm{ud}}, \kappa_{\mathrm{s}}\right)=(0.13770,0.13640)$. A red symbol denotes the result for the nonperturbative $v$.

where $v$ is adjusted such that the effective speed of light $c_{\text {eff }}$ becomes unity. Figure 1 shows an example of the nonperturbative tuning of $v$ with $\kappa_{\mathrm{h}}=0.11022$ at $\left(\kappa_{\mathrm{ud}}, \kappa_{\mathrm{s}}\right)=(0.13770,0.13640)$. In order to search for the physical charm quark mass point, we employ two values of the hopping parameter of a heavy quark $\kappa_{\mathrm{h}}$. The values of $\kappa_{\mathrm{h}}$ are chosen to sandwich the physical charm quark mass.

\section{Heavy-heavy system}

Let us first investigate the charmonium spectrum. At each combination of $\left(\kappa_{\mathrm{ud}}, \kappa_{\mathrm{s}}\right)$ we determine the physical point of the charm quark by the condition that the mass of the spin-averaged $1 S$ state reproduces the experimental value, $M(1 S)=\left(M_{\eta_{c}}+3 M_{J / \psi}\right) / 4=3.0677(3)[\mathrm{GeV}][9]$. For this purpose we linearly interpolate the results for $M(1 S)$ at two values of the hopping parameter $\kappa_{\mathrm{h}}$. Figure 2 illustrates this procedure for the case of $\left(\kappa_{\mathrm{ud}}, \kappa_{\mathrm{s}}\right)=(0.13770,0.13640)$.

In Fig. 3 we plot results for the mass of the orbital excitation $m_{\chi_{c 1}}(1 P)-m_{J / \psi}(1 S)$ at the physical point of the charm quark mass. It is hard to detect the dynamical quark mass dependence within the errors in the range of $3.5 \mathrm{MeV} \lesssim m_{\mathrm{ud}} \lesssim 12 \mathrm{MeV}$ and $73 \mathrm{MeV} \lesssim m_{\mathrm{s}} \lesssim 90 \mathrm{MeV}$. A very short chiral extrapolation is made employing a linear function of the up-down and the strange quark 


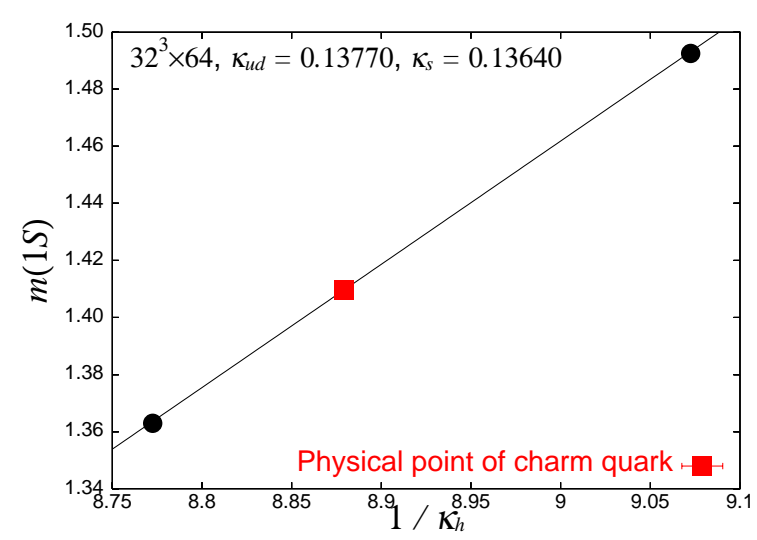

Figure 2: Interpolation of the spin-averaged $1 S$ charmonium mass to the physical point as a function of $\kappa_{\mathrm{h}}$. Errors are within symbols.

masses:

$$
m_{\chi_{c 1}}(1 P)-m_{J / \psi}(1 S)=\alpha+\beta m_{\mathrm{ud}}+\gamma m_{\mathrm{s}} .
$$

In Fig. 3 we find that the extrapolated value and its error are almost identical to the result at $\left(\kappa_{\mathrm{ud}}, \kappa_{\mathrm{s}}\right)=(0.137785,0.13640)$. This illustrates how close our simulation points are to the physical point. The result at the physical point is consistent with the experimental value within the error.

Figure 4 shows the results for the hyperfine splitting $m_{J / \psi}-m_{\eta_{c}}$. As in the orbital excitation we find little dynamical quark mass dependence. We extrapolate the results to the physical point employing a linear function of the dynamical quark masses. The extrapolated value, which is essentially determined by the result at $\left(\kappa_{\mathrm{ud}}, \kappa_{\mathrm{s}}\right)=(0.137785,0.13640)$, shows a $10 \%$ deficit from the experimental value. Possible sources of the discrepancy are $O\left(g^{2} a\right)$ effects in the relativistic heavy quark action, dynamical charm quark effects and disconnected loop contributions. A recent $2+1$ flavor lattice QCD calculation with the highly improved staggered quarks[10] shows a similar value to ours.

We compare our results for the hyperfine splitting in $N_{f}=2+1$ QCD with the previous $N_{f}=0,2$ results $[11,12]$ in Fig. 5. We observe a clear trend that the results become closer to the experimental value as the number of the flavor is increased. The dynamical quarks give significant contributions to the hyperfine splitting.

\section{Heavy-light system}

For the heavy-light system we focus on the $D$ and $D_{s}$ mesons and their decay constants measured at $\left(\kappa_{\mathrm{ud}}, \kappa_{\mathrm{s}}\right)=(0.137785,0.13660)$. This data point is so close to the physical point that $m_{\mathrm{ud}}$ corrections in the results could be smaller than the statistical errors. We employ perturbative values for the renormalization factor and the improvement coefficients of the axial vector current evaluated in Ref. [13]. For $c_{A_{4}}^{+}$we incorporate the nonperturbative contribution at the massless limit by $c_{A_{4}}^{+}=\left(c_{A_{4}}^{+}\left(m_{Q} a\right)-c_{A_{4}}^{+}(0)\right)^{\mathrm{PT}}+c_{A}^{\mathrm{NP}}$ with $c_{A}^{\mathrm{NP}}=-0.03876106[14]$. Figure 6 compares our results for the $D$ and $D_{s}$ meson masses with the experimental values [15]. They are consistent within 


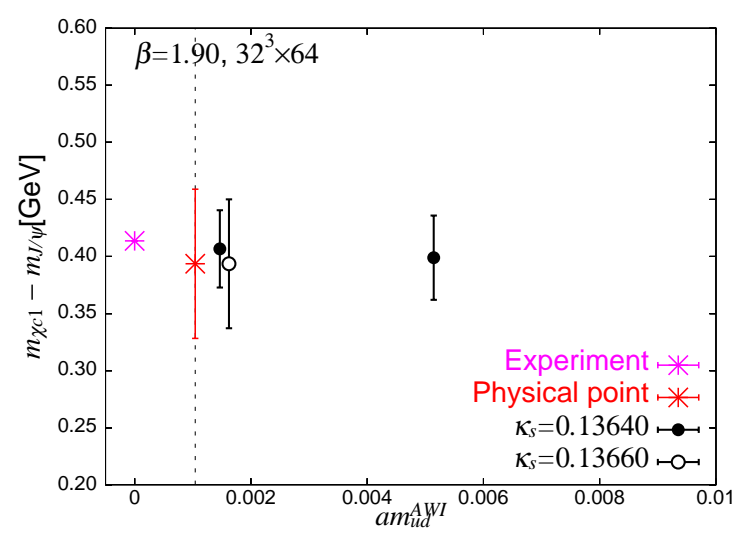

Figure 3: Orbital excitation $m_{\chi_{c 1}}-m_{J / \psi}$ as a function of $m_{\mathrm{ud}}^{A W I}$. Vertical dotted line denotes the physical point.

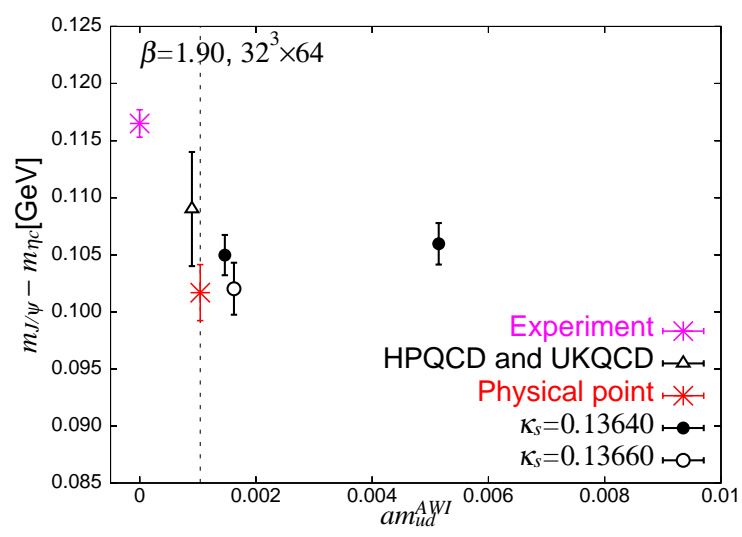

Figure 4: Hyperfine splitting of the charmonium as a function of $m_{\mathrm{ud}}^{A W I}$. A vertical dotted line denotes the physical point.

the errors. It is noteworthy that the physical charm quark mass determined from the heavy-heavy system successfully reproduces the heavy-light meson masses. The results for the decay constants are shown in Fig. 7, where we also plot the recent 2+1 flavor lattice QCD results with relativistic heavy quark actions[16, 17] for comparison. Although we find a sizable discrepancy between our result and the experimental value for $f_{D_{s}}$, we should analyze the full configuration set and improve statistics before we derive any conclusions.

In Fig. 8 we plot the ratios of $f_{D_{s}}$ to $f_{D}$ and $f_{D_{s}}$ to $f_{K}$ in which uncertainties coming from the perturbative renormalization factors and the lattice cutoff should cancel out. For both cases our results show larger values than the experimental ones, which originate from the discrepancy found in Fig. 7.

\section{Acknowledgments}

Numerical calculations for the present work have been carried out on the PACS-CS com- 


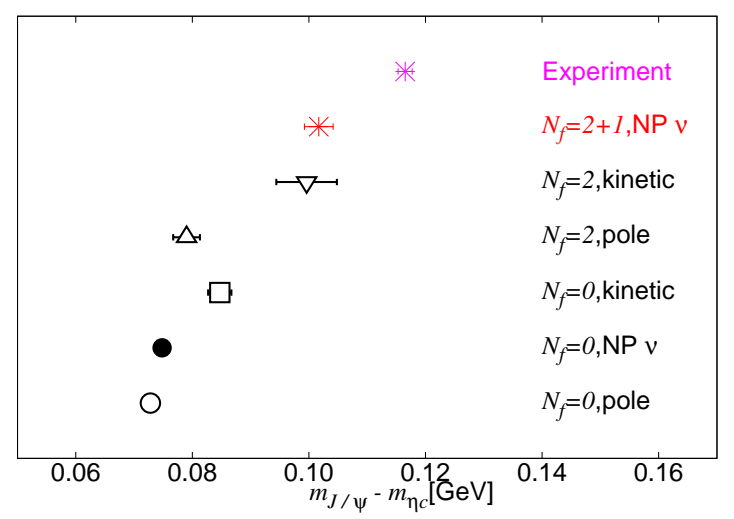

Figure 5: Comparison of hyperfine splittings in $N_{f}=0[11], 2[12]$ and 2 +1 , together with the experimental value. All the lattice results are obtained at $a^{-1} \approx 2 \mathrm{GeV}$.
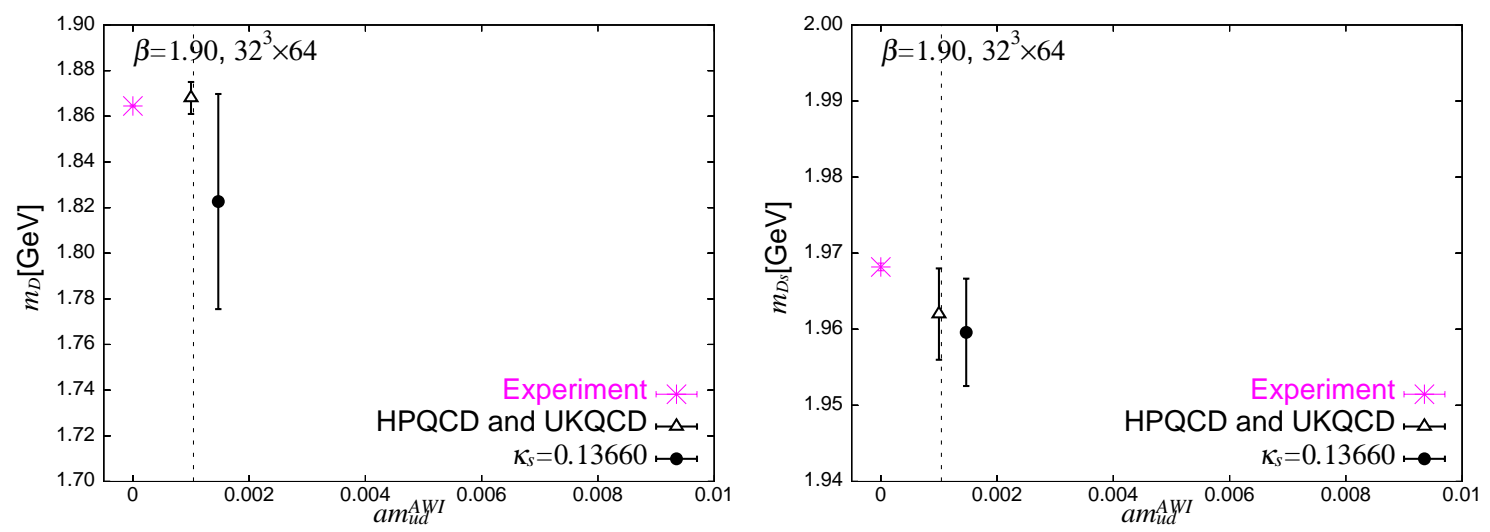

Figure 6: $D$ (left) and $D_{s}$ (right) meson masses as a function of $m_{\mathrm{ud}}^{\mathrm{AWI}}$. A vertical dotted line denotes the physical point.

puter under the "Interdisciplinary Computational Science Program" of Center for Computational Sciences, University of Tsukuba. This work is supported in part by Grants-in-Aid for Scientific Research from the Ministry of Education, Culture, Sports, Science and Technology (Nos. 16740147, 17340066, 18104005, 18540250, 18740130, 19740134, 20340047, 20540248, 20740123, 20740139 ) .

\section{References}

[1] For a review, see E. Gamiz, PoS (LATTICE 2008) 014.

[2] S. Aoki, Y. Kuramashi and S. Tominaga, Prog. Theor. Phys. 109 (2003) 383.

[3] CP-PACS and JLQCD Collaborations, S. Aoki et al., Phys. Rev. D73, 034501 (2006).

[4] PACS-CS Collaboration, S. Aoki et al., arXiv:0807.1661;

[5] Y. Kuramashi, PoS (LATTICE 2008) 018. 

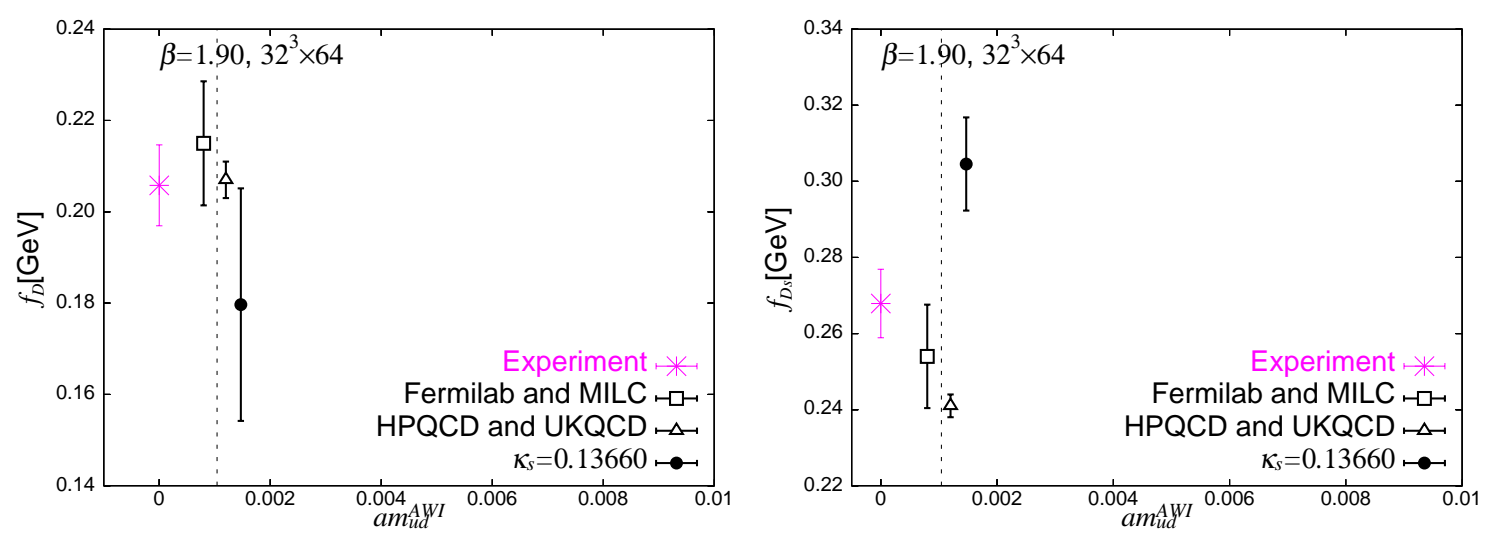

Figure 7: $f_{D}$ (left) and $f_{D_{s}}$ (right) meson masses as a function of $m_{\mathrm{ud}}^{\mathrm{AWI}}$. A vertical dotted line denotes the physical point.
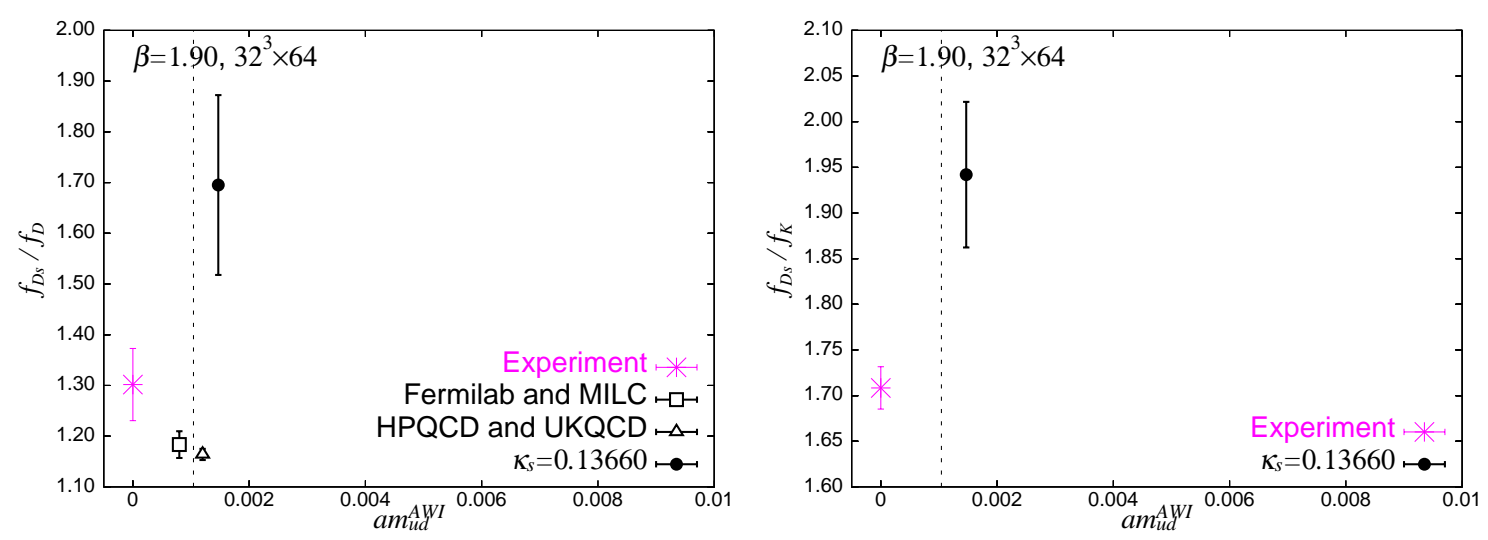

Figure 8: Ratios of $f_{D_{s}}$ to $f_{D}$ (left) and $f_{D_{s}}$ to $f_{K}$ (right). A vertical dotted line denotes the physical point.

[6] PACS-CS Collaboration, N. Ukita et al., PoS (LATTICE 2008 ) 097.

[7] PACS-CS Collaboration, D. Kadoh et al., PoS (LATTICE 2008 ) 092.

[8] S. Aoki, Y. Kayaba and Y. Kuramashi, Nucl. Phys. B 697 (2004) 271.

[9] C. Amsler et al., Phys. Let. B 667 (2008) 1.

[10] E. Follana et al., Phys. Rev. D75, 054502 (2007).

[11] CP-PACS Collaboration, Y. Kuramashi et al., PoS (LATTICE 2005 ) 226.

[12] CP-PACS Collaboration, Y. Kayaba et al., JHEP 0702 (2007) 019; Nucl.Phys.Proc.Suppl. 140 (2005) 479.

[13] S. Aoki, Y. Kayaba and Y. Kuramashi, Nucl. Phys. B 689 (2004) 127.

[14] CP-PACS/JLQCD and ALPHA Collaboration, T. Kaneko et al., JHEP 0704 (2007) 092.

[15] S. Stone, arXiv:0806.3921.

[16] HPQCD and UKQCD Collaborations, E. Follana et al., Phys. Rev. Lett. 100 (2008) 062002.

[17] FNAL Lattice, HPQCD and MILC Collaborations, C. Aubin et al., Phys. Rev. Lett. 95 (2005) 122002. 